\title{
Click chemistry approach to functionalize two-dimensional macromolecules of graphene oxide nanosheets
}

\section{Liang Kou, Hongkun He and Chao Gao*}

A facile "click chemistry" approach to functionalize 2D macromolecules of graphene oxide nanosheets with poly (ethylene glycol) of different molecular weights, polystyrene, palmitic acid and various amino acids was presented. FTIR, TGA, Raman spectroscopy, XPS, XRD, TEM, AFM and SEM were utilized to characterize the products. High degree of functionalization was achieved on the flat surfaces of graphene oxide, affording polymer-grafted 2D brushes and amino acids-immobilized nanosheets, which show improved solubility in organic solvents. The click chemistry strategy reported herein provides a facile and general method for functionalization of graphene oxide with macromolecules and desired biomolecules.

Keywords: Graphene; Click chemistry; Chemical modification; PEG; Amino acids

Citation: Liang Kou, Hongkun $\mathrm{He}$ and Chao Gao "Click chemistry approach to functionalize two-dimensional macromolecules of graphene oxide nanosheets”, Nano-Micro Lett. 2, 177-183 (2010). doi: 10.5101/nml.v2i3.p177-183

Graphene, a novel kind of two-dimensional (2D) macromolecule, has drawn tremendous attentions in the domain of scientific research. Due to its special properties of Young's modulus, thermal conductivity, mobility of charge carriers and specific surface area, it shows wide applications in energy-storage materials, "paper-like" materials, polymer composites and so on [1-3]. Graphene oxide (GO), derived from the oxidation of graphite, possesses abundant oxygen-containing functional groups, which not only render GO moderate water-dispersibility but also offer reactive sites for the further modification. Therefore, various reactions utilizing these oxygen-containing groups have been developed [4-8]. Haddon et al. have modified GO with long-chain alkylamine, making GO dispersed well in organic solvent [9]. Stankovich and coworkers functionalized GO with isocyanate derivatives and the resulting products show good solubility in polar aprotic organic solvents [10]. Chen et al. demonstrated that porphyrin and fullerene modified-graphene afforded the useful nonlinear optical properties [11]. In addition to small molecules, polymers were also employed to functionalize $\mathrm{GO}$ in order to improve the performance of $\mathrm{GO}$ in distinct domains. Yang et al. synthesized poly(2-(dimethylamino) ethyl methacrylate) (PDMAEMA)- grafted GO by ATRP [12], while Salavagione et al. functionalized GO with poly(vinyl alcohol) by esterification of carboxylic groups [13]. However, to functionalize GO with a facile and general strategy is still a challenge.

In addition, click chemistry, characterized by its modular nature, high selectivity and yields, has attained great development in recent years. Among various types of click reactions, the $\mathrm{Cu}$-catalyzed Huisgen 1, 3-dipolar cycloaddition of azides and alkynes plays a particularly important role in organic synthesis for its excellent performance such as stability against dimerization or hydrolysis [14]. Since the azide-alkyne click chemistry has been demonstrated as a useful tool to modify carbon nanotubes (CNTs) [15-21], it would be quite efficient for the functionalization of $\mathrm{GO}$, the analogue of CNTs. Most recently, the click chemistry has been tried to functionalize GO $[22,23]$. However, the reaction conditions and related influential

MOE Key Laboratory of Macromolecular Synthesis and Functionalization Department of Polymer Science and Engineering, Zhejiang University, 38 Zheda Road, Hangzhou 310027, P. R. China

*Corresponding author. E-mail: chaogao@zju.edu.cn 
factors have not been investigated. Since GO is a new type of atypical two dimensional (2D) macromolecules, it is vitally significant to evaluate the optimized "click" condition to modify its surface, in order to enlarge its application, especially in the areas of composites, multifunctional materials and devices. Herein, we employ azide-alkyne click chemistry to functionalize GO with linear polymers, various amino acids, aliphatic chains, and investigated the effect factors of reaction conditions.

\section{Experimental Part}

\section{Materials}

Graphite powder $(40 \mu \mathrm{m})$ was obtained from Qingdao Henglide Graphite Co., Ltd. DMF, $\mathrm{K}_{2} \mathrm{~S}_{2} \mathrm{O}_{8}, \mathrm{P}_{2} \mathrm{O}_{5}, \mathrm{KMnO}_{4}, 30 \%$ $\mathrm{H}_{2} \mathrm{O}_{2}$ and concentrated $\mathrm{H}_{2} \mathrm{SO}_{4}$ were purchased from Sinopharm Chemical Reagent Co., Ltd and used as received. $\mathrm{NH}_{2}\left(\mathrm{CH}_{2}\right)_{3} \mathrm{~N}_{3}$ and alkyne-terminated PEG, PS, C16, Gly and Phe were synthesized in our lab [18,20,24]. CuBr (Aldrich, 98\%) was obtained from Aldrich and purified according to the published procedures [25]. $N, N, N$ ', $N$ ", $N$ "'-pentamethyldiethylenetriamine (PMDETA, 99\%), N-hydroxysuccinimide (NHS, 98\%), $\mathrm{N}$-(3-dimethylaminopropyl)-N'-ethylcarbodiimide hydrochloride $(\mathrm{EDC} \cdot \mathrm{HCl}, 99 \%)$ were purchased from Alfa Aesar and used as received.

\section{Preparation of GO, GO-N 3 , GO-PEG.}

GO was synthesized from natural graphite powder and the specific process is presented in our published paper [26,27]. The water solution of $\mathrm{GO}(100 \mathrm{ml}, 7.5 \mathrm{mg} / \mathrm{ml})$ reacted with 3-azidopropan-1-amine $\left(\mathrm{NH}_{2}\left(\mathrm{CH}_{2}\right)_{3} \mathrm{~N}_{3}\right)(7.5 \mathrm{~g}, 75 \mathrm{mmol})$ for 24 $\mathrm{h}$ at room temperature under the catalytic action of EDC ( $4.79 \mathrm{~g}$, $25 \mathrm{mmol})$ and NHS (2.475 g, $22 \mathrm{mmol})$. The reaction product,
GO- $\mathrm{N}_{3}$, was centrifuged for $5 \mathrm{~min}$ at $1000 \mathrm{rpm}$ and washed five times with ultrapure water and then kept in water. Alkyne-terminated PEG (400 mg) was added into a round flask $(250 \mathrm{ml})$ containing GO-N $3(16 \mathrm{ml}, 2.5 \mathrm{mg} / \mathrm{ml})$, and then DMF (144 ml), CuBr (200 mg, $1.3 \mathrm{mmol})$, PMDETA (294 $\mu \mathrm{l})$ were added to the flask under the atmosphere of $\mathrm{N}_{2}$. The coupling reaction between azide and alkyne proceeded for $150 \mathrm{~min}$ at $60^{\circ} \mathrm{C}$ in an oil bath. Then, the reaction product was centrifuged for $10 \mathrm{~min}$ at $5000 \mathrm{rpm}$ and washed five times with DMF. All products were dried in a vacuum oven at $60^{\circ} \mathrm{C}$ overnight, and then grinded them into powder for the use of analytical measurement.

\section{Measurements}

Thermogravimetric analysis (TGA) was carried out on a Perkin-Elmer Pyris 6 TGA instrument under nitrogen with a heating rate of $20^{\circ} \mathrm{C} / \mathrm{min}$. Infrared spectra (IR) were recorded on BRUKER VECTOR 22 spectrometer. Scanning electron microscope (SEM) images were measured by Hitachi S4800 field-emission SEM system. Raman measurements were carried out using a LabRam-1B Raman spectroscope equipped with a $632.8 \mathrm{~nm}$ laser source. X-ray Diffraction (XRD) was carried out in X'Pert PRO diffractometer equipped with $\mathrm{Cu} \mathrm{K} \alpha$ radiation (40 $\mathrm{kV}, 40 \mathrm{~mA}$ ). Atomic force microscopy (AFM) was done using a Digital Instrument Nanoscope IIIa scanning probe microscope, operating at the tapping mode, with samples prepared by spin-coating sample solutions onto freshly cleaved mica substrates at $2500 \mathrm{rpm}$. X-ray photoelectron spectroscopy (XPS) were performed with a RBD upgraded PHI-5000C ESCA system (Perkin-Elmer) with $\mathrm{Mg} \mathrm{K} \alpha$ radiation $(\mathrm{h} v=1253.6 \mathrm{eV}$ ) at a power of $250 \mathrm{~W}$. Transmission electron microscope (TEM) was performed in FEI-PHILLIPS CM 200 electron microscope

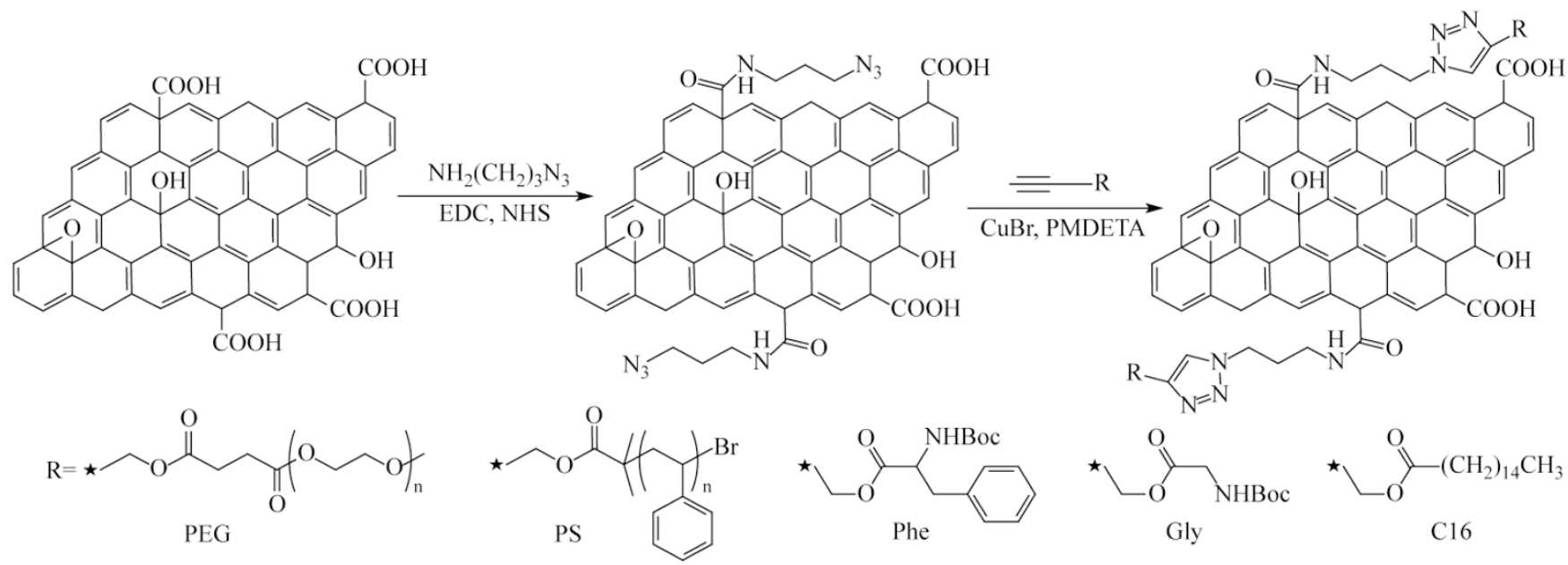

Scheme 1. Functionalization of 2D macromolecules of GO nanosheets by azide-alkyne click chemistry. 
operating at $160 \mathrm{kV}$.

\section{Results and Discussion}

Our functionalization protocol is shown in Scheme 1. Carboxylic groups of GO react with 3-azidopropan-1-amine $\left(\mathrm{NH}_{2}\left(\mathrm{CH}_{2}\right)_{3} \mathrm{~N}_{3}\right)$ to form GO-N $\mathrm{N}_{3}$ by EDC condensation. Subsequently, alkyne-terminated poly(ethylene glycol) (PEG) with different molecular weight, polystyrene (PS), propargyl alcohol-esterified palmitic acid (C16), glycin (Gly) and phenylalanine (Phe), react with azide groups via click chemistry, affording GO-PEG, GO-PS, GO-C16, GO-Gly and GO-Phe, respectively.

Typically, the case of PEG was selected to show the results of click chemistry in details and the molecular weight of PEG is 750 unless specially labelled. Figure 1A shows the FTIR spectra of GO, GO-N 3 and GO-PEG. The peaks at 1720 and $1626 \mathrm{~cm}^{-1}$ can be assigned to the stretching of carboxyl groups and the aromatic $\mathrm{C}=\mathrm{C}$ stretching, respectively. After the EDC condensation, azide groups were attached to the surfaces of GO, which could be confirmed from the appearance of the characteristic IR absorption peak of azide groups at $2098 \mathrm{~cm}^{-1}$. For GO-PEG, the disappearance of the peak at $2098 \mathrm{~cm}^{-1}$ and the presence of the peak at $1100 \mathrm{~cm}^{-1}$ related to the stretching of $\mathrm{C}-\mathrm{O}$ groups of PEG indicated the successful azide-alkyne coupling reaction on the nanosheets.

To explore the effect of reaction conditions on the nanoscale click coupling, we traced the reaction process by FTIR measurements at 25,40 and $60^{\circ} \mathrm{C}$, by taking out part of the reaction solution at a given time on the basis of extremely sensitive azido absorption in FTIR spectrum (see Fig. 1B). With the peak at $1626 \mathrm{~cm}^{-1}$ as an internal standard, the integration ratio of the peaks at 2098 and $1626 \mathrm{~cm}^{-1}\left(I_{\mathrm{N} 3} / I_{\mathrm{G}}\right)$ represents the degree of the click conversion. Generally, the value of $I_{\mathrm{N} 3} / I_{\mathrm{G}}$ decreases drastically at first and then quite slowly until almost zero (see Fig. $1 \mathrm{C})$, indicating that the reaction rate is very fast in the beginning but quite slow in the end. Moreover, the temperature has significant influence on the click reaction. A $c a$. $93.0 \%$ conversion was achieved within 30,60 , and $900 \mathrm{~min}$ at 60,40 , and $25^{\circ} \mathrm{C}$, respectively. In other words, 68.6, 93.3 and $98.8 \%$ conversions were found at the same reaction time of $60 \mathrm{~min}$ for cases of 25,40 and $60^{\circ} \mathrm{C}$, respectively. In our previous study, the click coupling reached equilibrium on CNTs at around $8 \mathrm{~h}$ [20]. Herein, the elevated click rate on GO is likely attributed to the fact that both faces of GO can be readily attacked while CNTs can be only accessed on their outer surfaces.

Accordingly, $60^{\circ} \mathrm{C}$ was chosen as the temperature to complete other click reactions (see Scheme 1). Interestingly, the azido peak at $2098 \mathrm{~cm}^{-1}$ cannot be detected in the corresponding FTIR spectrum after $150 \mathrm{~min}$ in all cases except GO-PS in which
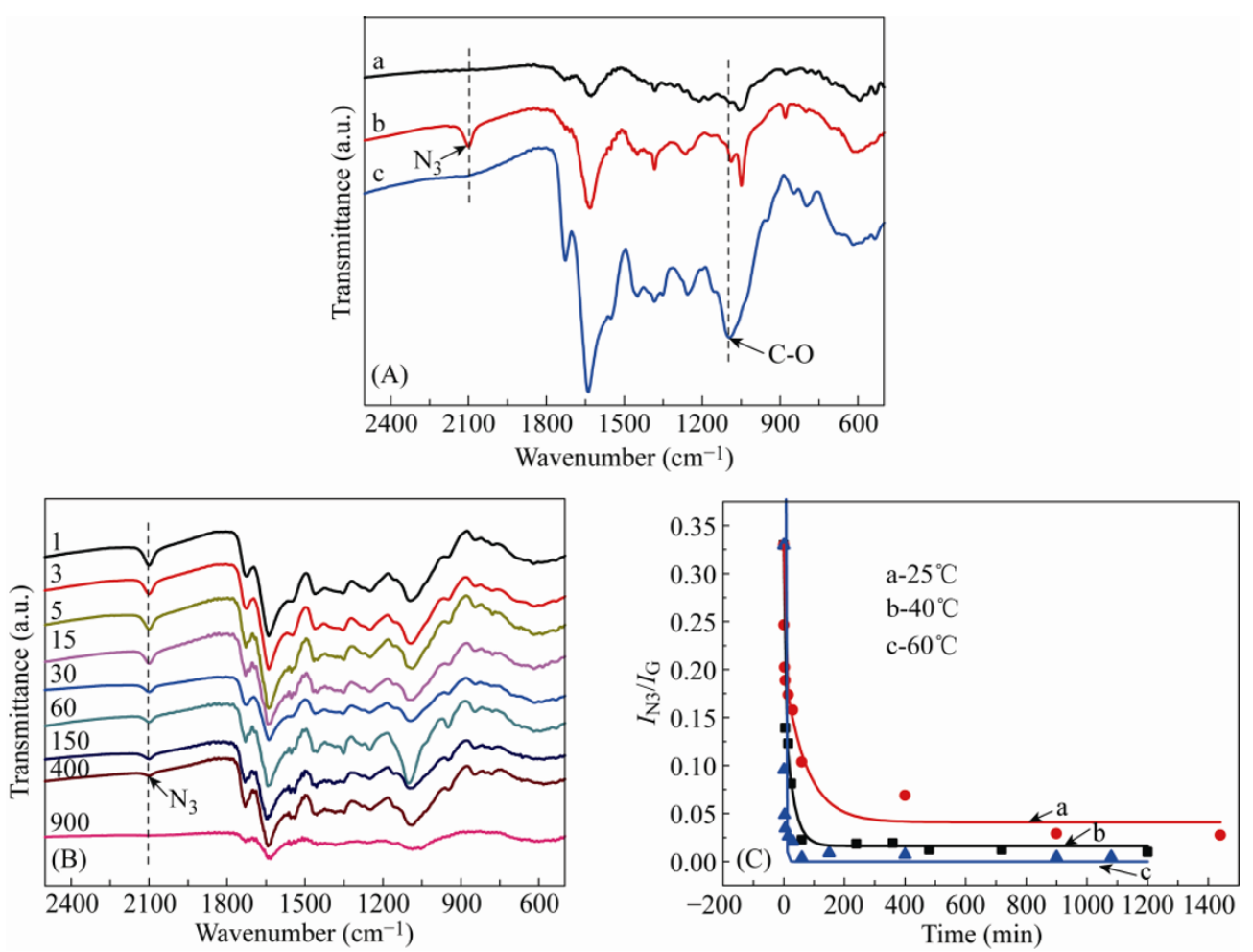

FIG. 1. (A) FTIR spectra of GO (a), GO-N 3 (b) and GO-PEG (c); (B) FTIR spectra of GO-PEG at different reaction time at $25^{\circ} \mathrm{C}$ (from 1 to 900 min); (C) Conversion of azido group for the synthesis of GO-PEG at different reaction time and temperature. 

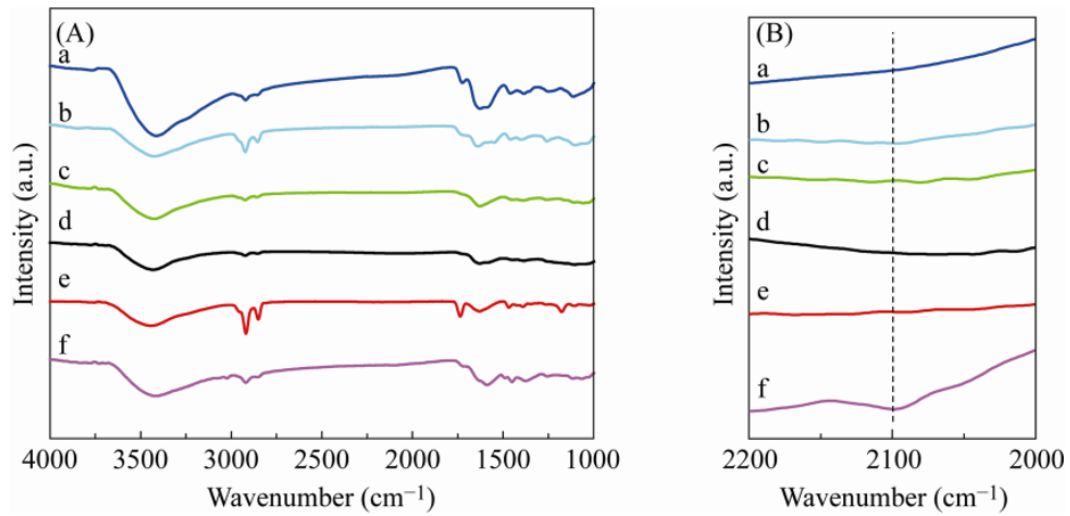

FIG. 2. FTIR spectra of GO-PEG (350) (a), GO-PEG (1900) (b), GO-Gly (c), GO-Phe (d), GO-C16 (e) and GO-PS (f): (A) region between 1000 and $4000 \mathrm{~cm}^{-1}$; (B) Expanded region between 2000 and $2200 \mathrm{~cm}^{-1}$, highlighting the residual azide stretch at $2100 \mathrm{~cm}^{-1}$.

the azido peak can be still slightly observed (see Fig. 2). It is shown that the "graft-to" click efficiency is affected by two main factors under the same reaction conditions: the molecular weight and flexibility (or chemical structure) of reactants [28]. In our system, all of alkyne-terminated PEG with different molecular weights (including 350, 750 and $1900 \mathrm{~g} / \mathrm{mol}$ ) reacted with azide groups of GO-N $\mathrm{N}_{3}$ efficiently for the disappearing of azido peak within $150 \mathrm{~min}$. This suggests that molecular weight of this kind of flexible and "thinner" polymer has little effect on the click coupling on the flat nanosurface of GO sheets. Compared with alkyne-terminated PEG (1900), alkyne- terminated PS with a comparable molecular weight $(\mathrm{Mn}=2500 \mathrm{~g} / \mathrm{mol})$ is much more rigid and "thicker", resulting in stronger steric hindrance effect and much lower contacting (or click) probability between rigid nanosheets and PS chains. This result is similar to our previous investigation that two hard nanoparticles are difficult to be clicked together except a soft interlayer exists [19]. Nevertheless, small rigid molecules such as phenylalanine can be easily immobilized to GO-N $\mathrm{N}_{3}$ nanosheets for its high contacting chance associated with high mobility, casting affirmative light on the click fabrication of multifunctional biomolecules-conjugated graphene.

TGA was further employed to confirm the successful click reactions on the surfaces of GO. It should be noted that the larger weight than $100 \%$ of samples is the result of $\mathrm{N}_{2}$ absorption during the measurement. As shown in Fig. 3, the whole weight loss of GO is $c a .51 .0 \%$ and nearly all weight loss occurs at $c a$. $210{ }^{\circ} \mathrm{C}$, which is presumably due to pyrolysis of the labile oxygen-containing functional groups. It was thought that the weight loss should increase after EDC condensation because of the attachment of azide moieties, but in fact the weight loss is decreased to $39.1 \%$ due to the partial reduction of GO during the reaction, which can be proved from our XRD datum (see Fig.
3D). In addition, the changed color of reaction solution from brown to dark also indicates the process of reduction. The weight loss of $15.8 \%$ at $c a .210^{\circ} \mathrm{C}$ can be ascribed to the pyrolysis of the residual labile oxygen-containing functional groups while the weight loss of $23.3 \%$ between 210 and $500^{\circ} \mathrm{C}$ is due to the pyrolysis of azide moieties. For GO-PEG, on account of the polymer decomposition, the increased weight loss of $52.4 \%$ was observed. Calculated from corresponding TGA weight loss data, the content of PEG molecules on the surfaces of GO is $c a .0 .17$ mmol per gram of GO-PEG, corresponding to one PEG chain per 250 carbon atoms. We also traced the click reaction by TGA and found that the weight loss of GO-PEG at $3 \mathrm{~min}$ at $60^{\circ} \mathrm{C}$ was almost equal to that at one hour at $25^{\circ} \mathrm{C}$ (Figure S2 in Supporting Information), further confirming the much faster click reaction at elevated temperature. In addition, the TGA of GO-Phe is also performed and presented in Fig. S6, showing the similar result to the PEG case.

Raman spectroscopy is a powerful approach to investigate the structural and electronic properties of graphene. As shown in Fig. 3B, the pristine graphite is characterized by a strong band at $\sim 1575 \mathrm{~cm}^{-1}$ ( $G$ band) which corresponds to an $\mathrm{E}_{2 \mathrm{~g}}$ mode of graphite and a weak band at $\sim 1330 \mathrm{~cm}^{-1}$ ( $D$ band) related to the vibration of carbon atoms with dangling bonds in plane terminations of disordered graphite. After the process of oxidation, GO shows a broadened $G$ band shifted to $1595 \mathrm{~cm}^{-1}$ and a broadened $D$ band with high intensity at $1330 \mathrm{~cm}^{-1}$. The intensity ratio of the $D$ and $G$ bands $\left(I_{\mathrm{D}} / I_{\mathrm{G}}\right)$ is commonly used to evaluate the quality of carbon materials. The ratio of $I_{\mathrm{D}} / I_{\mathrm{G}}$ increases gradually from natural graphite $(0.403)$ to GO (1.234), to $\mathrm{GO}^{-\mathrm{N}_{3}}$ (1.397) and then to GO-PEG (1.399), revealing the increased degree of functionalization that is quite similar to the case of CNTs [29]. Meanwhile, we also obtained Raman spectra 

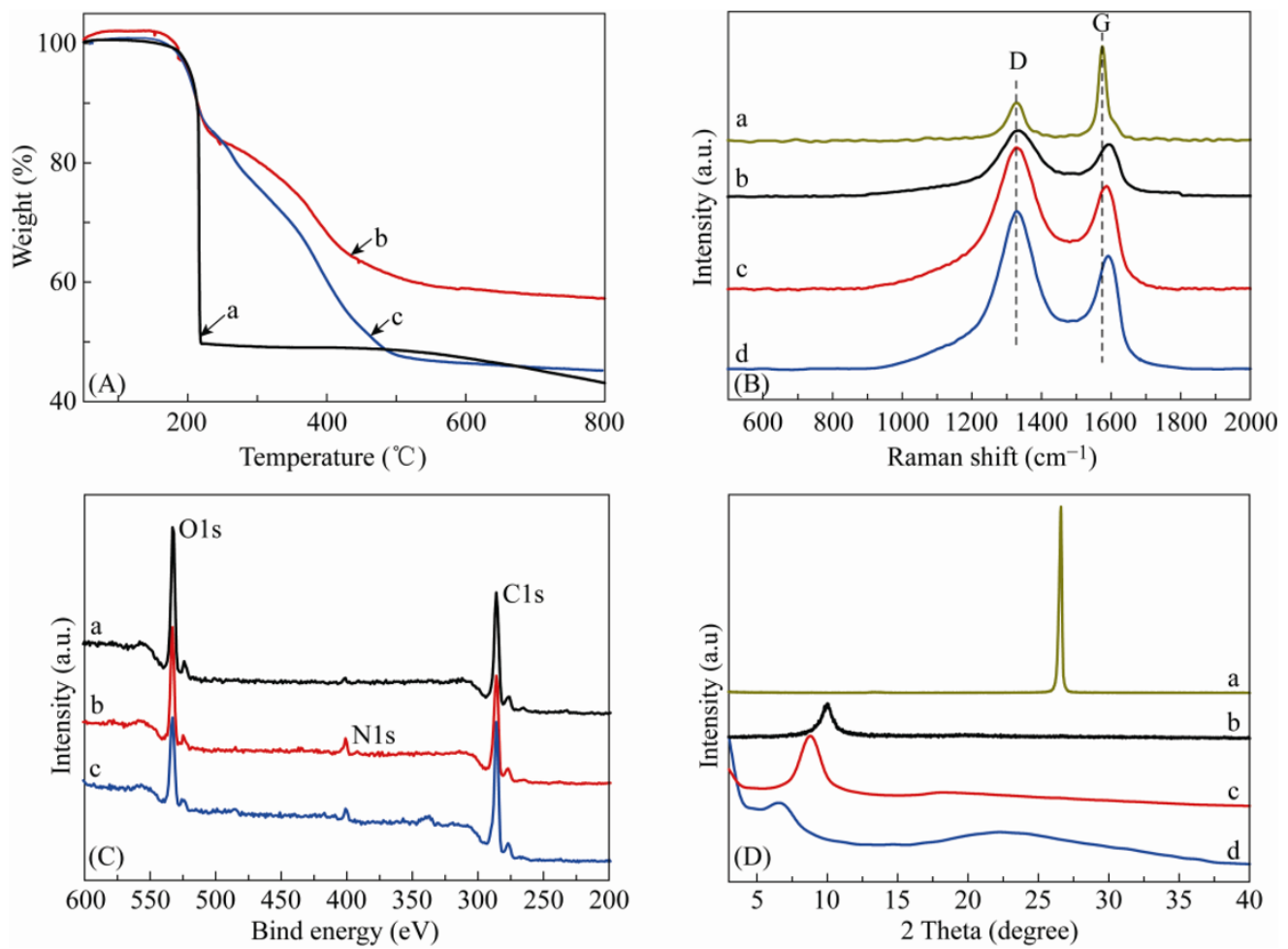

FIG. 3. (A) TGA weight loss curves of GO (a), GO-N 3 (b) and GO-PEG (c); (B) Raman spectra of graphite (a), GO (b), GO-N 3 (c) and GO-PEG (d); (C) XPS spectra of GO (a), GO-N 3 (b) and GO-PEG (c); (D) XRD patterns of graphite (a), GO (b), GO-N $\mathrm{N}_{3}$ (c), GO-PEG (d).

of GO-C16, GO-Gly and GO-Phe, and the value of $I_{\mathrm{D} /} I_{\mathrm{G}}$ is 1.404 , 1.455 and 1.486, respectively (see Fig. S5).

XPS was used to study surface elemental composition of different specimens. Figure 3C shows the XPS spectra of GO, GO-N ${ }_{3}$ and GO-PEG. The nitrogen content increased from 1.2\% for $\mathrm{GO}$ (nitrogen absorption from atmosphere) to $4.9 \%$ for GO- $\mathrm{N}_{3}$ and then decreased to $3.8 \%$ for GO-PEG, confirming the successful attachment of azide groups and PEG. In addition, the carbon content of GO-C16 is as high as $80.9 \%$ compared with the content of $65.8 \%$ for $\mathrm{GO}-\mathrm{N}_{3}$, which also proves the accomplishment of the click coupling reaction (Table S1 in Supporting Information).

XRD measurement is also performed to further study the changes in structure. As shown in Fig. 3D, the initial graphite powder shows the typical sharp diffraction peak at $2 \theta=26.5^{\circ}$, with the corresponding d-spacing of $0.336 \mathrm{~nm}$. For GO, no
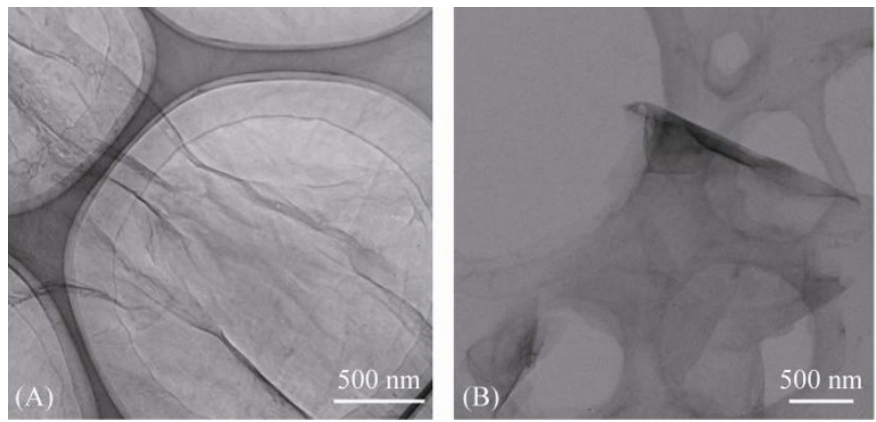

diffraction peaks from the parent graphite material is observed, but a new broad peak at $2 \theta=10.0^{\circ}$ appears, with a d-spacing of $0.885 \mathrm{~nm}$. The increase of the d-spacing is due to the decoration with epoxide and hydroxyl groups intercalated between the GO layers during the oxidation process and a conversion of hybridization of the oxidized carbon atoms from sp2 to sp3 [30]. Due to the introduction of 3-azidopropan-1-amine, GO- $\mathrm{N}_{3}$ shows a diffraction peak at $2 \theta=8.8^{\circ}$, corresponding to a d-spacing of $1.005 \mathrm{~nm}$. In addition, GO- $\mathrm{N}_{3}$ exhibits a new diffraction peak at $2 \theta=18.8^{\circ}$, demonstrating the partial reduction of $\mathrm{GO}-\mathrm{N}_{3}$ during the reaction process. For GO-PEG, the left shift diffraction peak at $2 \theta=6.6^{\circ}$, corresponding to a d-spacing of $1.339 \mathrm{~nm}$, can be ascribed to the attachment of PEG chains. What is more, GO-PEG suffer a further reduction for the diffraction peak at $2 \theta=22.4^{\circ}$.

TEM analyses of GO and GO-PEG were performed by
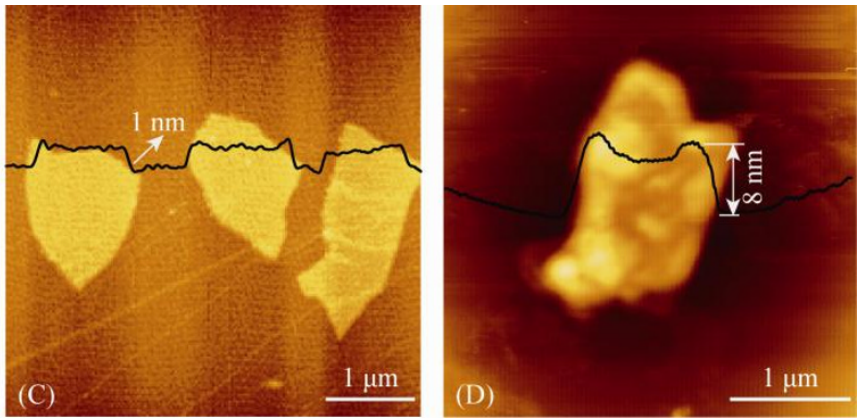

FIG. 4. TEM images of GO (A) and GO-PEG (B). AFM images of GO (C) and GO-PEG (D). 


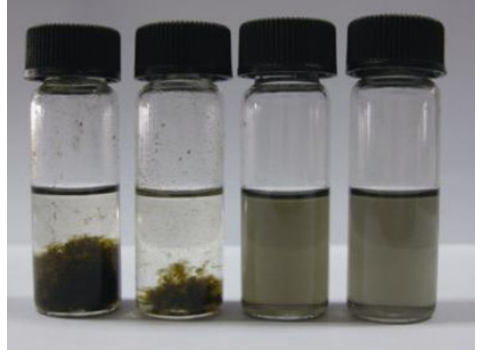

FIG. 5. Photographs of GO and GO-PEG placed in solvents (from left to right: GO in dichloromethane, GO in chloroform, GO-PEG in dichloromethane and GO-PEG in chloroform)

placing a single drop of the DMF solution onto a holey carbon-coated copper grid. As shown in Fig. 4A, 4B, GO displays a morphology like a large crumpled paper while the edge of GO-PEG scrolls just as a tube, similar with the previous study which reported that the polymer-coated graphene scrolls and folds above the polymer's glass transition temperature [31]. AFM is a direct and reliable method to examine the degree of exfoliation of GO to graphene single sheets. The as-prepared GO and GO-PEG sheets were characterized by tapping mode AFM and the results are shown in Fig. 4C, 4D. The average thickness of GO nanosheets is $c a$. $1 \mathrm{~nm}$, which indicates a completed exfoliation to be single-layer nanosheets, and lateral dimensions of $c a .1 \mu \mathrm{m}$ are observed. For GO-PEG, the surface topography become more complicated and the thickness increased to $c a .8$ $\mathrm{nm}$, which demonstrates the successful attachment of PEG. Moreover, the saddleback-like height profile of GO-PEG illustrates that more carboxylic groups locate on the edge of GO nanosheets.

In addition, the final products including GO-PEG show improved solubility in a variety of solvents such as chloroform, dichloromethane and so on (see Fig. 5), which will make considerable contribution to the application of graphene in the area of composites and other aspects.

\section{Conclusions}

In summary, an efficient click chemistry approach to functionalize 2D macromolecules of graphene oxide nanosheets has been presented and demonstrated with linear polymers and various amino acids. High degree of functionalization was achieved on the flat surfaces of GO, affording polymer-grafted $2 \mathrm{D}$ brushes and amino acids-immobilized nanosheets. The click chemistry strategy opens the door for facile functionalization of GO with macromolecules and desired biomolecules. The relevant work is in progress and will be reported later.
This work was financially supported by the National Natural Science Foundation of China (No. 50773038, and No. 20974093), National Basic Research Program of China (973 Program) (No. 2007CB936000), Qianjiang Talent Foundation of Zhejiang Province (2010R10021), the Fundamental Research Funds for the Central Universities (2009QNA4040), and the Foundation for the Author of National Excellent Doctoral Dissertation of China (No. 200527).

Electronic Supplementary Information (ESI) available: [SEM of GO and GO-PEG; TGA of GO-PEG at different reaction time, IR, XRD, XPS, Raman of GO-Gly, GO-Phe, and GO-C16, TGA of GO-Phe].

Received 13 August 2010; accepted 13 September 2010; published online 29 September 2010.

\section{References}

1. S. Park and R. S. Ruoff, Nat. Nanotech. 4, 217 (2009). doi:10.1038/nnano.2009.58

2. A. K. Geim and K. S. Novoselov, Nat. Mater. 6, 183 (2007). doi:10.1038/nmat1849

3. C. N. R. Rao, A. K. Sood, K. S. Subrahmanyam and A. Govindaraj, Angew. Chem. Int. Ed. 48, 7752 (2009). doi:10.1002/anie.200901678

4. D. R. Dreyer, S. Park, C. W. Bielawski and R. S. Ruoff, Chem. Soc. Rev. 39, 228 (2010). doi:10.1039/b917103g

5. J. F. Shen, Y. Z. Hu, C. Li, C. Qin and M. X. Ye, Small 5, 82 (2009). doi:10.1002/smll.200800988

6. X. Y. Yang, X. Y. Zhang, Y. F. Ma, Y. Huang, Y. S. Wang and Y. S. Chen, J. Mater. Chem. 19, 2710 (2009). doi:10.1039/b821416f

7. S. J. Park, K. S. Lee, G. Bozoklu, W. W. Cai, S. T. Nguyen and R. S. Ruoff, ACS Nano 2, 572 (2008). doi:10.1021/nn700349a

8. S. J. Park, D. A. Dikin, S. T. Nguyen and R. S. Ruoff, J. Phys. Chem. C 113, 15801 (2009). doi:10.1021/jp907613s

9. S. Niyogi, E. Bekyarova, M. E. Itkis, J. L. Mcwilliams, M. A. Hamon and R. C. Haddon, J. Am. Chem. Soc. 128, 7720 (2006). doi:10.1021/ja060680r

10. S. Stankovich, R. Piner, S. T. Nguyen and R. S. Ruoff, Carbon 44, 3342 (2006). doi:10.1016/j.carbon.2006. $\underline{06.004}$ 
11. Z. B. Liu, Y. F. Xu, X. Y. Zhang, X. L. Zhang, Y. S. Chen and J. G. Tian, J. Phys. Chem. B 113, 9681 (2009). doi:10.1021/jp9004357

12. Y. F. Yang, J. Wang, J. Zhang, J. C. Liu, X. L. Yang and H. Y. Zhao, Langmuir 25, 11808 (2009). doi:10.1021/ $\underline{\text { la901441p }}$

13. H. J. Salavagione, M. A. Gómez and G. Martínez, Macromolecules 42, 6331 (2009). doi:10.1021/ma9008 $\underline{45 \mathrm{~W}}$

14. H. C. Kolb, M. G. Finn and K. B. Sharpless, Angew. Chem. Int. Ed. 40, 2004 (2001). doi:10.1002/ 1521-3773(20010601)40:11<2004::AID-ANIE2004>3.0. $\mathrm{CO} ; 2-5$

15. H. M. Li, F. O. Cheng, A. M. Duft and A. Adronov, J. Am. Chem. Soc. 127, 14518 (2005). doi:10.1021/ja054958b

16. G. K. Such, J. F. Quinn, A. Quinn, E. Tjipto and F. Caruso, J. Am. Chem. Soc. 128, 9318 (2006). doi:10.1021/ $\mathrm{ja} 063043+$

17. S. Campidelli, B. Ballesteros, A. Filoramo, D. D. Diaz, G. de la Torre, T. Torres, G. M. A. Rahman, C. Ehli, D. Kiessling, F. Werner, V. Sgobba, D. M. Guldi, C. Cioffi, M. Prato and J. P. Bourgoin, J. Am. Chem. Soc. 130, 11503 (2008). doi:10.1021/ja8033262

18. Y. Zhang, H. K. He and C. Gao, Macromolecules 41, 9581 (2008). doi:10.1021/ma801696z

19. H. K. He, Y. Zhang, C. Gao and J. Y. Wu, Chem. Commun. 1655 (2009). doi:10.1039/b821280e

20. Y. Zhang, H. K. He, C. Gao and J. Y. Wu, Langmuir 25, 5814 (2009). doi:10.1021/la803906s
21. J. Han, and C. Gao, Nano-Micro Lett. 2, 2010, In press, online publication: http://nmletters.org/index.php/nml/ article/view/107

22. R. Salvio, S. Krabbenborg, W. J. M. Naber, A. H. Velders, D. N. Reinhoudt and W. G. van der Wiel, Chem. Eur. J. 15, 8235 (2009) · doi:10.1002/chem.200900661

23. S. T. Sun, Y. W. Cao, J. C. Feng and P. Y. Wu, J. Mater. Chem. 20, 5605 (2010). doi:10.1039/c0jm01269f

24. C. Gao, H. K. He, L. Zhou, X. Zheng and Y. Zhang, Chem. Mater. 21, 360 (2009). doi:10.1021/cm802704c

25. G. L. Cheng, A. Boker, M. F. Zhang, G. Krausch and A. H. E. Muller, Macromolecules 34, 6883 (2001). doi:10.1021/ma0013962

26. Z. Xu and C. Gao, Macromolecules 43, 6716 (2010). doi:10.1021/ma1009337

27. H. K. He and C. Gao, Chem. Mater. 22, 5054 (2010). doi: $10.1021 / \mathrm{cm} 101634 \mathrm{k}$

28. H. F. Gao and K. Matyjaszewski, J. Am. Chem. Soc. 129, 6633 (2007). doi:10.1021/ja0711617

29. C. Gao, Y. Z. Jin, H. Kong, R. L. D. Whitby, S. F. A. Acquah, G. Y. Chen, H. H. Qian, A. Hartschuh, S. R. P. Silva, S. Henley, P. Fearon, H. W. Kroto and D. R. M. Walton, J. Phys. Chem. B 109, 11925 (2005). doi: 10.1021/jp051642h

30. M. J. McAllister, J. L. LiO, D. H. Adamson, H. C. Schniepp, A. A. Abdala, J. Liu, M. Herrera-Alonso, D. L. Milius, R. CarO and R. K. Prud'homme, Chem. Mater. 19, 4396 (2007). doi:10.1021/cm0630800

31. Q. Li, Z. J. Li, M. Chen and Y. Fang, Nano Lett. 9, 2129 (2009). doi:10.1021/n1900681n 